\title{
Proxemic Interaction Applied to Public Screen in Lab
}

\author{
Huiliang Jin, Tao Xu, Bertrand David, and René Chalon \\ Université de Lyon, CNRS \\ Ecole Centrale de LYON, LIRIS, UMR5205 \\ \{huiliang.jin, tao.xu, bertrand. david, rene. chalon\} @ec-lyon. fr
}

\begin{abstract}
Proxemics is the terminology used to describe spatial relationships among humans while communicating with each other. It could be interesting to apply the proxemics theory to the domain of human computer interaction, namely proxemic interaction. Computers, unlike people, find it hard to interpret instantly and precisely the user's nonverbal hints, such as body postures, movement, and distance. With the development of computer vision, these tasks can be performed with simple devices. In this paper, we build the abstract model for calculation in proxemic interaction, and further illustrate the prototype based on research life in the lab. We then describe evolution of the prototype through investigation of proxemic interaction. Finally, we ask users for their opinion via a preliminary user study and usability test. Our study shows that users are attracted by this kind of interaction, and especially by the application scenario in the lab with a large public screen.
\end{abstract}

Keywords: Proxemics, Proxemic interaction, Public screen, implicit interaction.

\section{Introduction}

Proxemics is a psychological concept, which is nonverbal terminology. It is defined as "the interrelated observations and theories of man's use of space as a specialized elaboration of culture". Edward T. Hall [4] identifies two overreaching categories, personal space and territory. He divides the space around a person into four categories - intimate, personal, social and public space, though these partitions could vary according to culture. It should be mentioned that proxemics rely not only on culture but on gender. Researchers found that men encroach frequently on a female's space, while the female is reluctant to "violate" a man's personal space [6]. In recent years, proxemics has been transplanted to artificial intelligence, ubiquitous computing and human computer interactions $[2,3,5,7,8]$. As a development of ubiquitous computing and computer vision, it is now possible to make computers measure the user's spatial relationship by means of a camera, passive markers or other technologies. Proxemic Interaction (PI) normally considers five factors: distance, orientation, movement, identity of entities, and location of feature [2]. Distance and movement imply the entity's (in this case the user's) intention of interaction. Orientation implies the user's focus of attention. Identity and location are useful contextual information. Location refers to layout of the fixed or semi-fixed features around the proxemics interaction 
environment. We could explore many interesting interaction modes with single factor or multi-factor combinations, in particular with the application relating to a public screen [8], as a public screen could be deemed a "virtual" user rather than a passive machine. In this way, we could communicate with a public screen implicitly and naturally, just like we would communicate with another person, instead of explicitly and rigidly commanding "operation" of a machine. A public screen mounted in the public space of the lab (such as a coffee room) or bus shelter is a practical way of exploring PI usability. As there is a diversity of users with different genders, cultures and ages.

We first build the PI model based on a single detector. Then we describe the PI scenario with a public screen in a research lab, and present our prototypes. We end the paper with an analysis for usability tests and our proposal for future work.

\section{User's Behavior Model}

As aforementioned, PI needs to consider at least five factors. Among them, distance, orientation and movement are base sets to calculate user's proxemics relative to a public screen equipped with a detector. In this case, we abstract 3-dimensional coordinates (Fig. 1): (0, YC, 0) are the coordinates of the screen equipped with detector, $\mathrm{YC}$ is the detector height, and (XU, YU, ZU) are the user's coordinates, where YU is the user height. This abstraction is not fine-grained, as it abstracts both user and screen as points. Nevertheless, it is sufficient to calculate the three factors for the spatial relationship. If there is more than one user, we could also add them as points, and calculate their relative positions.
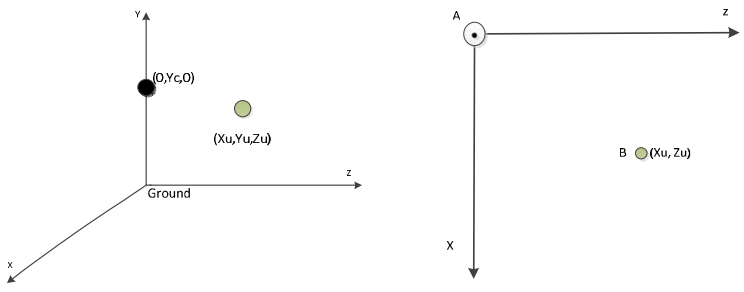

Fig. 1. PI coordinates

The user's movement in front of the screen is continuous. To track the user's movement, we change the user's coordinates from $\left(X_{U}, Y_{U}, Z_{U}\right)$ to $\left(X_{U}, Y_{U}, Z_{U}, T_{U}\right)$ where $\left\{T_{U}\right\}$ is the user's time point in one position.

For the same user, $\left\{\mathrm{Y}_{\mathrm{U}}\right\}$ does not change during interaction. It could be simplified as $\left(\mathrm{X}_{\mathrm{U}}, \mathrm{Z}_{\mathrm{U}}, \mathrm{T}_{\mathrm{U}}\right)$ : we pick up three points every 2 seconds, then obtain a vector $\overrightarrow{A B}=$ $\left(\mathrm{X}_{\mathrm{U}}, \mathrm{Z}_{\mathrm{U}}\right)$, in which case $\|\overrightarrow{A B}\|=\sqrt{X_{U}^{2}+Z_{U}^{2}}$ is the accurate distance of user to screen. Then, with time as one parameter, $\overrightarrow{A B_{t}}=\left(\mathrm{X}_{\mathrm{Ut}}, \mathrm{Z}_{\mathrm{Ut}}, \mathrm{t}\right)$,

$$
\overrightarrow{\left\|A B_{x}\right\|}=\sqrt{X_{U x}^{2}+Y_{U x}^{2}}, \mathrm{x} \in\{\mathrm{t}, \mathrm{t}+2, \mathrm{t}+4\}
$$

If $\left\|\overrightarrow{A B_{t+4}}\right\|-\left\|\overrightarrow{A B_{t+2}}\right\|>\mu,\left\|\overrightarrow{A B_{t+2}}\right\|-\left\|\overrightarrow{A B_{t}}\right\|>\mu$, the user is walking away from the screen, If $\left\|\overrightarrow{A B_{t}}\right\|-\left\|\overrightarrow{A B_{t+2}}\right\|>\mu,\left\|\overrightarrow{A B_{t+2}}\right\|-\left\|\overrightarrow{A B_{t+4}}\right\|>\mu$, the user is 
approaching. Where $\mu$ is a threshold, if it is a small value, PI will be more nimble, otherwise it will be more blunt. With this model, we could calculate the user's position instantly and predict the user's potential movements. However, we do not take orientation into account. For PI with a single screen, orientation is not always necessary.

\section{Scenario of Proxemic Interaction on a Public Screen Deployed in a Research Lab}

We build an application based on professional life in a research lab (Fig. 2). Just as in our lab, there is a diversity of users, with students from China and France, as well as many potential users from different countries as visitors.

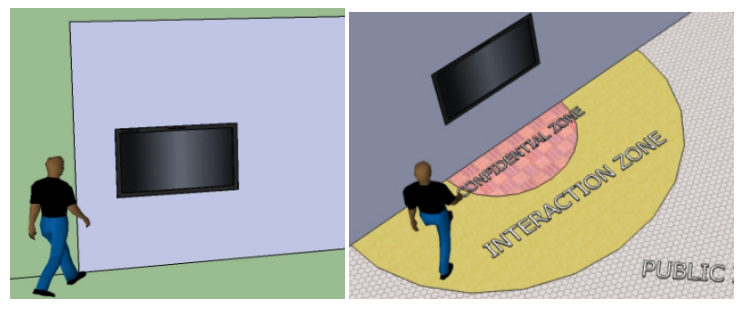

Fig. 2. A public screen in the lab

We divided the space in front of the screen into three partitions, as follows:

- Public zone (PZ): users in this zone can always view public information at a glimpse while passing by. If they want to check details, they will enter next zone;

- Interaction zone (IZ): users in this zone are recognized, and the screen displays limited personal information. E.g. it displays "Hello, Mr. Andrew" instead of "Hello, Jack Andrew": this trick attracts users and meanwhile protecting privacy;

- Confidential zone (CZ): users in this zone obtain a part of the screen which is strictly reserved for them (by choice), i.e. personal display space (PDS). This space displays personal information that the user authorizes to display, and the PDS moves along with the user in front of the screen.

This zone classification is not strict. If we are more concerned with privacy, we could add another zone between IZ and CZ, and vice versa. Except for the proximity features, the screen should also include some other features as follows:

- It is networked. Each member of the lab could update the personal contents via their own computers, and customize the personal information to be displayed.

- It is multi-partition. It could thus be used simultaneously by several users publicly and privately, as well as with simultaneous implicit and explicit interaction.

- It supports multi-person collaborative interaction. Users in CZ involved in collaboration work could easily exchange resources with one another. 
Since the screen offers ambient and contextual awareness, we identified six questions to guide interaction design, known as $5 \mathrm{~W}[1] 1 \mathrm{H}$ :

1. WHO, who engages in interaction?

2. WHEN, when does the user arrive? And how long does he stay?

3. WHERE, where is the user positioned near the screen?

4. WHAT, what does the user want?

5. WHY, why do users adopt that kind of behavior? Interpret user's activities.

6. HOW, How multi-user interact collaborative with each other? In fact, as we will consider interaction with mobile devices in the future, we need to clarify how users interact with or without mobile devices.

To study how to take the six factors into account, based on discussion in the lab and user study we identified five typical scenarios (Table 1)

Table 1. Scenario Classification

\begin{tabular}{|c|c|c|c|}
\hline & Morning & Noon & Evening \\
\hline Single User & 1 & 2 & 3 \\
\hline Single User in CZ with Others waiting in IZ & \multicolumn{3}{|c|}{5} \\
\hline Multi-user collaborative interaction in CZ & \multicolumn{3}{|c|}{} \\
\hline
\end{tabular}

Scenario 1: a user named Jack ANDREW comes to the lab in the morning, and goes to take a coffee. When he enters the coffee room, he can see the general message from the screen hung on the wall, such as:

- Recent conference call for paper;

- Lab news, such as new publications, lectures or meetings;

- Technology news, related to the domain;

- Status of laboratory members, such as attendance status;

- New ideas shared by others for brainstorming.

Then, if he is interested by the new idea shared by another student, with his coffee, he steps into IZ. More details about each message will be displayed. Meanwhile the screen rapidly recognizes him, and pops up a banner at the bottom of the screen saying "Good Morning, Mr. ANDREW", along with some thumbnails of his personal messages, such as, "you have 7 new e-mails and 1 forthcoming conference".

If he just wants to check the new idea, then he waves this out by gestures, and raises his hand to select and browse details. One moment later, he finishes his coffee, and if he just walks away, the screen will return to the neutral state. Otherwise, if he thinks the idea is fairly interesting and wants to add some comments, then he steps further into the confidential zone.

Once he steps into $\mathrm{CZ}$, a dialog box pops up to ask him whether he wants to be assigned a PDS. If he selects YES, all his personal information that he authorized will be displayed in PDS. Meanwhile, the other parts of the screen continue to be reserved for public information. If he chooses NO, some available operations menus arise 
surround the "idea" Textbox. He adds comments and sends it to the proposer. Afterwards, he turns back and leaves, and the screen resumes its neutral state.

Scenario 2: Jack comes back at noon, it is now lunch time. He doesn't want to eat in the university canteen today. He decides to eat outside with some friends. He walks directly to the screen IZ, after analyzing his personal tags, "I like sushi, and pasta", then compares with his history: for example, if he ate in an Italian restaurant last time, the screen displays the Japanese restaurant as priority, with the discount information. He could wave his hands to select. Then if he wants to check details, he could just step into CZ, and the details of the selected restaurant would be displayed. He could drag the details into his PDS, and then once he logs in his personal account with his mobile phone, he could view the details, such as the address and telephone number, without bothering to take a photo of the map, or noting anything down. He could invite others by dragging this information to their icons on the screen sidebar.

Scenario 3: Jack finishes his job and wants to leave at $6 \mathrm{pm}$, he comes back to the screen, and stands in IZ. According to the time, the system infers that he wants to leave for home. Then it checks his history: if he prefers to take the bus every day, it will display the time of the next bus in large text. If he has a car, then the screen will display the traffic information on the map instead of the bus. He does not need to enter the $\mathrm{CZ}$, unless he wants to check the detailed bus timetable. Before leaving, he wants to make an appointment with a professor. So he steps into $\mathrm{CZ}$ and clicks the professor's icon, checking when he is available. He just needs to select one idle time, and the screen saves the appointment request automatically. He could also write some remarks and then send them. The professor can read the message once he is in the screen IZ, or he could check this by email as well.

Scenario 4: If Jack is in interaction in CZ, while other users are approaching and waiting in IZ, the size of the PDS assigned to Jack will be reduced to protect privacy, and also save more space for other people receiving messages.

Scenario 5: Multi-users refer to two or more users in CZ. With respect to the lab's practical situation, there are several possibilities according to user roles:

- Two visitors: they are not recognized, so the screen displays public info for them;

- Two members of the lab, who are not familiar friends. This could be inferred by the distance between them. As strangers tend to stand separately. The screen thus assigns two PDSs relatively far from each other in order to respect their privacy.

- Two members of the lab, who are friends. Contrary to the last situation, these two will stand close together, and thus have two PDSs displayed close to each other. Users could also select to merge their PDSs, for easy sharing of some contents.

- One member and one visitor: the screen just displays info related to the member, ranging from general to personal. The visitor will only see the public message.

\section{Prototype Evolution of the PI Public Screen in the Lab}

We have designed and developed two prototypes: one paper prototype and another low-fidelity PI public screen prototype. 


\section{PI paper prototype.}

This kind of prototype is used to gather users' suggestions prior to development, and for primary usability tests. It is mainly aimed at UI which is displayed to users when they are in CZ. As shown in Figure 3, a is UI when two users who do not know each other stand in CZ simultaneously. On the contrary, b is UI for two users who are well acquainted. Their PDSs are merged for sharing. The UI layout is in large text and graphic. The sidebar contains a list of members in the lab: if they are in the lab, their image will be lightened, if not their image is grey. Urgent messages such as "CALL FOR PAPER" are highlighted in red.

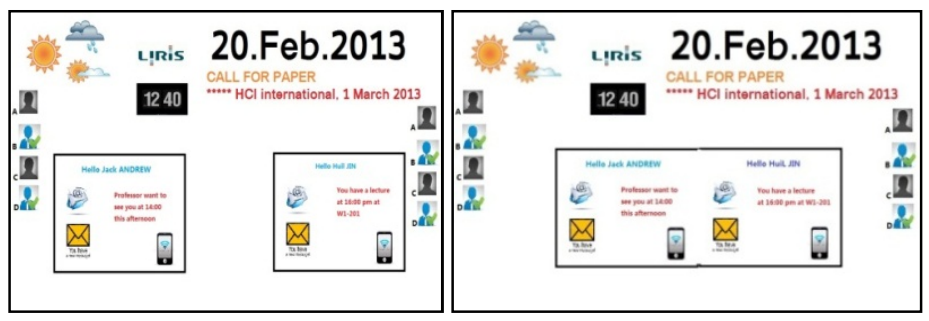

Fig. 3. Paper prototypes for the PI public screen (a b)

2. Double screen prototype.

We simulate two screens (left and right) installed in the airport or railroad station as information boards (Figure 4a). This prototype uses one camera to recognize the user's id, and by ARtoolkit markers to calculate user's distance and orientation to screen. Normally, the information board in the airport displays all the departure flights. Passengers need to run a detailed search to find their flight. In this prototype, when a user equipped with ARToolkit markers approaches this screen sufficiently close, it will only display his flight. And if he turns left, then the next screen located on the left will display the same information, and vice versa. If there are more than two users present, both screens display general flight information to protect privacy. We also designed another interface (Figure $4 b$ ). This is an overview of a user's agenda and a proximity bar, which indicates the user's distance from the screen.
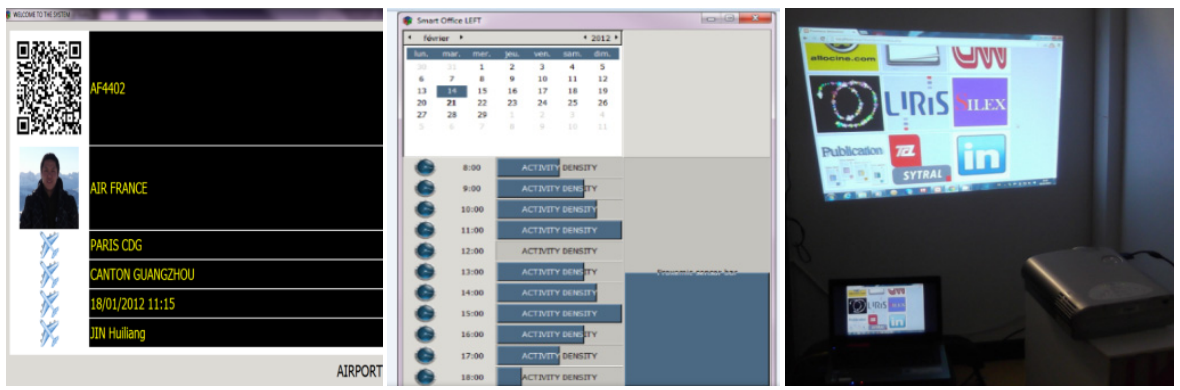

Fig. 4. Low fidelity prototype (from left to right a b c) 


\section{Single screen prototype.}

Based on the first two prototypes, we found that ARToolkit markers are not ideal for detecting the user's position, as they are extremely sensitive to light conditions. Some testers are annoyed by the additional markers. Also, a two-screen prototype is not necessary for the lab for the time being. As a result, we have reduced the prototype to one screen (Fig 4c), optimized the framework, and designed a Web UI (Fig 5).
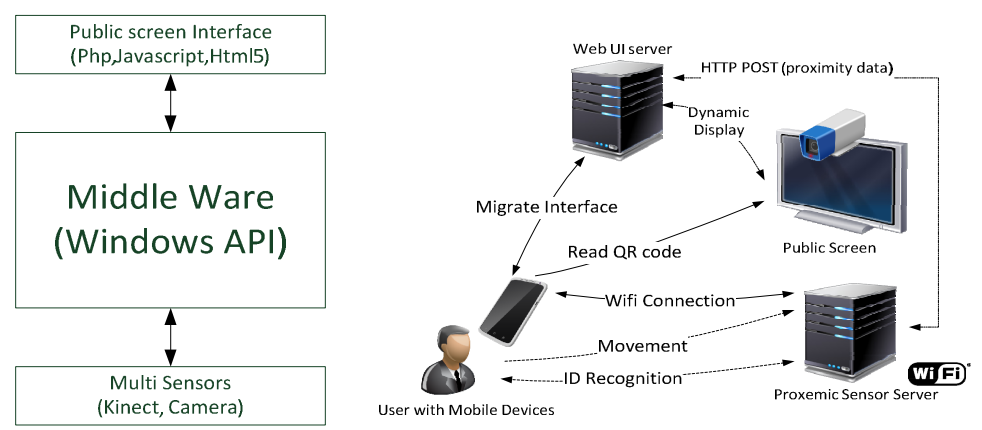

Fig. 5. Framework of the PI public screen (a b)

Figure 5a shows the overall structure of the prototype: we use two sensors, kinect for user position measurement, and camera for ID recognition by face. The Windows API is middleware which processes data acquired from sensors, and converts them to operation commands. Figure $5 \mathrm{~b}$ shows workflows for single user interaction, mobile devices interact and backstage processing, distinguished by line pattern. UML sequence diagram of two users (correspondence to scenario 5) as illustrated in Figure 6.

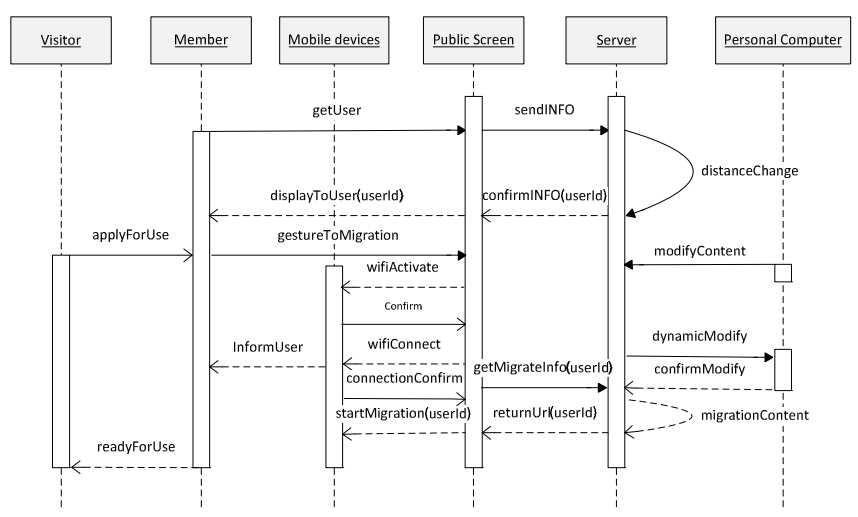

Fig. 6. Two users' interaction Sequence Diagram in UML 
The prototype defines three interaction modalities by three aforementioned zones.

- Users in PZ could only see general information from the projected screen, and they will not be recognized.

- Users in IZ could be recognized if they are members of the lab, and both members and visitors could interact with the screen by gestures, up to 8 types. Authorized users operate the UI elements easily, as illustrated in figure 7. It should be noted that the same gestures made by users in different zones imply different operations, e.g. if users in CZ stretch out their arms, this implies zooming out the UI, while if users in IZ make the same gesture, this implies zooming in the UI. Also, if the user is editing a presentation file in a public screen (like PPT), if he moves backwards from IZ to PZ, the presentation will be automatically played, and the user could control the play sequence by waving his arm to the left or right. Then, if the user returns to the $\mathrm{CZ}$, the presentation will exit the play mode, and allow the user to edit it manually.

- Users in $\mathrm{CZ}$ are allowed to interact directly with the screen. Users in this zone could acquire their PDSs. Also, the screen will return the user's personal contents to him. For this aspect, we do not develop a high-fidelity prototype. We combine the paper prototype and graphical Wizard-of -Oz prototyping to illustrate how it works.

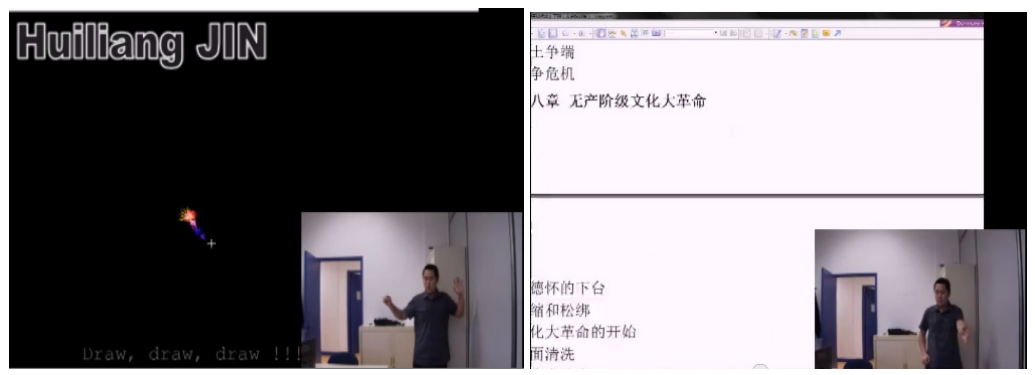

Fig. 7. Proxemics gesture interaction in IZ

\section{$5 \quad$ Usability Test and Results Analysis}

Once the first stage of the prototype was developed, we organized usability tests. We invited 10 volunteers, 3 of which are engineering students from our school, 4 are Chinese PhD students in the HCI domain, and 1 is a student from another university who is learning French. 1 student is from another city in France, who is also a PhD student but in a very different field: she is invited to conduct a remote user study. The last student is a French PhD student from our lab. Age ranges from 20 to 29, with 6 men and 4 women.

Our test is not dedicated to test robustness of the low-fidelity prototype, but rather to test usability and novelty, as these generate useful feedback for further research. We designed three test steps as follows, 
1. First, we explained the idea of PI to testers, and narrated the scenario: they are allowed to interrupt and ask questions, their questions are recorded.

2. Secondly, the user steps into IZ, and his/her ID is recognized. The system displays his/her name on the screen, after which the user is required to experience the PI by a simple game, i.e. via movement to control the cartoon character in the game, forward and backward. Then, the user is asked to browse the publication paper of the lab, and zoom in or out to change the size of the text, and adapt it for optimum comfort.

3. Lastly, the user stands in CZ, and interacts explicitly with the screen. While interaction with the touch screen is expected in the high-fidelity prototype, this is merely a primary usability test. Hence we merely ask the user to give commands, while the experimenter acting as a wizard simulates computer interaction with the paper prototype, and the UI shifts along with the user's movement in front of the screen. Additionally, we design an adaptive UI, allowing users to migrate UI to their device by reading QR code. Users could experience the UI migration from the public screen to their mobile devices.

On completion of the test, we asked the users to fill out a questionnaire. From Figure 8, we can conclude that our prototype is good at usability, as users are impressed by the PI: someone said "it's cool to control a computer from such a distance". And they also satisfied by the rapid response to their movement. However, they always complain about ID recognition, as it too easily recognizes them as another person. Also, when they try to migrate UI to their devices, the steps are somewhat tedious. Opinions vary as well: for example, one user said it's more convenient for their devices to connect to the public screen automatically with no code, while another user said he was worried about leaking his personal info by connecting to the public WiFi Hotspot even there is a code.

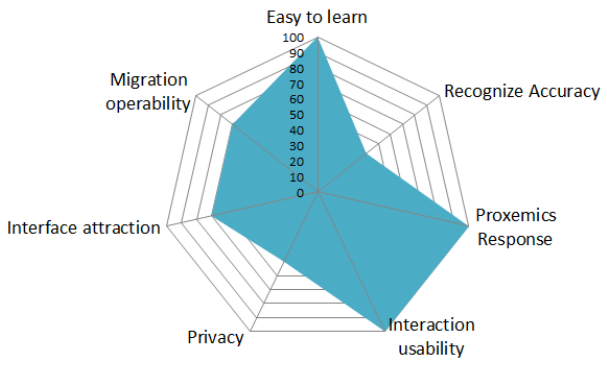

Fig. 8. Usability Test result

In addition to Figure 8, we also collected many useful suggestions from remote user studies and records during testing. We selected several suggestions as follows:

- Gestures should be natural: for example, some testers are not used to scrolling down the text using the "stretch out arm" gesture, as the feeling of rolling down something is more like waving hands down rather than raising the arm straight forward. 
- The design needs to avoid maloperation: for example, when the user moves his/ her hand trying to select an UI element, if his/her arm is across the center of the body, it could trigger another action by error.

- The prototype should offer some contents for entertainment, especially when the user comes in at noon or the evening, as he/she might be tired of work and just want to find something for relaxing from the screen.

\section{Conclusion and Future Work}

In this paper, we described Proxemic Interaction and built the user's behavior model, explaining how to analyze users' proxemic behavior while interacting with public screens. We propose that gender and emotion be considered as factors of PI study, as these are two key signs when people communicate with each other. We then apply the PI to the public screen, aiming at the research life in the lab. This is an attractive and practical topic, as well as a good means of exploring the application of this novel interaction. Through user studies and usability tests, we found that users are curious about this kind of interaction, and they made many useful suggestions. With respect to future work, we will consider possible ways of creating gender difference PIs and personalizing interaction modals to men and women, as well as PIs with consideration of emotion differences. By computer vision, to determine users' moods from their expression or even wear color, and finally design entire multi-user collaborative interaction modalities, along with development of high-fidelity prototype and usability tests.

\section{References}

1. Abowd, G.D., Mynatt, E.D.: Charting Past, Present, and Future Research in Ubiquitous Computing. ACM Transactions on Computer-Human Interaction (TOCHI) 7(1), 29-58 (2000)

2. Greenberg, S., Marquardt, N., Ballendat, T., Diaz-Marino, R., Wang, M.: Prox-emic Interactions: The New Ubicomp? Interactions 18(1), 42-50 (2011)

3. Harrison, C., Dey, A.K.: Lean and Zoom: Proximity-aware User Interface and Content Magnification. In: Proceedings of the Twenty-sixth Annual SIGCHI Conference on Human Factors in Computing Systems, pp. 507-510 (2008)

4. Hall, E.T.: The Hidden Dimension. Anchor Books (1966) ISBN 0-385-08476-5

5. Ju, W., Lee, B.A., Klemmer, S.R.: Range: Exploring Implicit Interaction Through Electronic Whiteboard Design. In: Proceedings of the 2008 ACM Conference on Computer Supported Cooperative Work, pp. 17-26 (2008)

6. Madden, S.J.: Proxemics and Gender: Where's the Spatial Gap. North Dakota Journal of Speech \& Theatre 12, 41-46 (1999)

7. Marquardt, N., Greenberg, S.: Informing the Design of Proxemic Iteractions. IEEE Pervasive Computing 11(2), 14-23 (2012)

8. Vogel, D., Balakrishnan, R.: Interactive Public Ambient Displays: Transitioning from Implicit to Explicit, Public to Personal, Interaction with Multiple Users. In: Proceedings of the 17th Annual ACM Symposium on User Interface Software and Technology, UIST 2004, pp. 137-146. ACM, New York (2004)

9. Xu, T., Jin, H., David, B., Chalon, R., Zhou, Y.: A Context-aware Middleware for Interaction Devices Deployment in AmI. In: HCI International 2013, Las Vegas (2013) 